\title{
Decreased sL-selectin serum levels in sleep apnea syndrome patients with cardiovascular diseases
}

\author{
Monika Kosacka ${ }^{A-F}$, Irena Porębska ${ }^{B-F}$, Renata Jankowska ${ }^{E, F}$ \\ Department of Pulmonology and Lung Cancer, Wroclaw Medical University, Poland \\ A - research concept and design; $\mathrm{B}$ - collection and/or assembly of data; $\mathrm{C}$ - data analysis and interpretation; \\ $D$ - writing the article; $E$ - critical revision of the article; $F$ - final approval of article
}

\section{Address for correspondence}

Monika Kosacka

E-mail:mokka113@hotmail.com

\section{Funding sources}

None declared

\section{Conflict of interest}

None declared

Received on June 29, 2015

Revised on December 31, 2015

Accepted on March 16, 2016

\begin{abstract}
Background. Obstructive sleep apnea syndrome (OSA) is a common disorder associated with an increased risk of cardiovascular diseases.

Objectives. SL-selectin is an adhesion molecule released from the surface of leukocytes as they are activated and may inhibit leukocyte attachment to the endothelium. The aim of this study was to evaluate sL-selectin serum levels in OSA patients with cardiovascular diseases.
\end{abstract}

Material and methods. A total of 163 OSA patients were enrolled in the study. The mean age was 55.41 \pm 8.63 years and the mean AHI (apnea hypopnea index) was $35.02 \pm 22.28 / \mathrm{h}$. A control group was composed of 59 healthy subjects. All subjects underwent a nocturnal respiratory polygraphy. SL-selectin serum levels were measured using the enzyme-linked immunosorbent assay (ELISA) method.

Results. sL-selectin serum levels were significantly lower in OSA patients than in the control group $(1080.02 \pm 175.29$ vs $1350.73 \pm 569.75 \mathrm{ng} / \mathrm{mL}, \mathrm{p}<0.05)$. In addition, there was a negative correlation between sL-selectin levels and AHI and DI and a positive correlation between sL-selectin levels and mean and minimum saturation. SL-selectin levels were lower in OSA patients with cardiovascular diseases than in those without co-morbidities. We also found that sL-selectin correlated positively with HDL-cholesterol (high density lipoprotein) and negatively with uric acid and CRP (C-reactive protein).

Conclusions. Our work, together with observations relating to other diseases and experimental studies, suggests that lower sL-selectin levels could play a role in an increased risk of cardiovascular complications in sleep apnea syndrome. However future studies are needed to understand the role of sL-selectin in sleep apnea syndrome.

Key words: cardiovascular risk, sleep apnea, P-selectin

DOI

$10.17219 /$ acem/62215

\section{Copyright}

Copyright by Author(s)

This is an article distributed under the terms of the

Creative Commons Attribution Non-Commercial License

(http://creativecommons.org/licenses/by-nc-nd/4.0/) 


\section{Introduction}

The relationship between obstructive sleep apnea syndrome (OSA) and cardiovascular disease is an interesting one. OSA increases the risk of hypertension, myocardial infarction and stroke, and has an influence on insulin resistance, obesity and metabolic changes. ${ }^{1,2}$ Many mechanisms may be helpful in explaining this association with the most important being sympathetic activity, oxidative stress, inflammation and endothelial dysfunction. ${ }^{2-4}$ It has also been suggested that OSA-induced hypoxic stress may contribute to cardiovascular diseases through the activation of adhesion molecules. ${ }^{5}$

L-selectin is a member of the selectin family. ${ }^{6}$ This family of adhesion molecules includes 3 receptors expressed from various cells including endothelial cells (E-selectin), leukocytes (L-selectin) to platelets and endothelium cells (P-selectin). ${ }^{7}$ L-selectin is expressed by granulocytes, lymphocytes and monocytes. It plays an important role in the launch of the adhesion cascade and in the capture and rolling of leukocytes along the endothelial cells. ${ }^{6,8} \mathrm{~L}$-selectin is susceptible to proteolytic cleavage and may exist in the blood stream in soluble form (sL-selectin). It is released from the surface of neutrophils as they are activated and is a marker of neutrophil activation. L-selectin inhibits leukocyte attachment to the endothelium by occupying receptors and in high concentration it may block adhesion completely. ${ }^{9}$

The aim of this study was to evaluate sL-selectin serum levels in OSA patients with cardiovascular diseases.

\section{Material and methods}

\section{Patients}

A total of 163 patients with newly diagnosed OSA were enrolled in the study. None of the patients examined had previously received OSA treatment. The group examined comprised 124 males and 39 females. The mean age was $55.41 \pm 8.63$ years. The mean AHI (apnea hypopnea index) was $35.02 \pm 22.28 / \mathrm{h}$. The majority of subjects were overweight and obese with mean BMI (body mass index) $34.98 \pm 7.55$. Many cardiovascular comorbidities were observed in the OSA patients. 113 patients had hypertension, 44 diabetes, 36 ischemic heart disease and 10 had suffered a stroke. The majority of patients with OSA had cardiovascular disease $(75 \%, \mathrm{n}=121)$. All patients with comorbidities received standard treatment according to international recommendations. In the majority of cases, antihypertensive treatment included an ACEI (angiotensin converting enzyme inhibitor), usually in combination with drugs from different groups. Five patients with ischemic heart disease had medical history of CABG (coronary artery bypass graft). All of the diabetes patients were treated with oral hypoglycemic agents. We divided the OSA patients into 2 groups: those with cardiovascular diseases $(n=121)$ and those without $(n=42)$. The first group included patients with one or more cardiovascular diseases. Only 42 of the patients presented OSA without any other cardiovascular disease.

A control group constituted 59 healthy subjects, including 28 females. All subjects from the control group underwent respiratory polygraphy tests. In addition, daytime sleepiness was not observed in any subjects in the control group. The mean age in the control group was $51.27 \pm 12.97$ years and the mean BMI was $29.47 \pm 5.42$.

\section{Polygraphy}

All patients and all subjects from the control group underwent a nocturnal respiratory polygraphy using a Grass Aura Lite PSG (Warwick, USA). The following parameters were evaluated during $8 \mathrm{~h}$ of nocturnal sleep: AHI, desaturation index - DI, mean and minimum $\mathrm{SaO}_{2}$ at the end of sleep apnea/hypopnea episodes. Apnea was defined as the cessation of airflow for more than $10 \mathrm{~s}$ and hypopnea as a reduction in airflow by at least $30 \%$ compared with its value during wakefulness for at least $10 \mathrm{~s}$ followed by a $4 \%$ or greater decrease in oxyhemoglobin saturation. An oxygen desaturation event was detected when oxygen saturation fell by at least $4 \%$. In all cases, manual scoring was carried out after automatic scoring.

\section{Selectin}

Blood samples were collected from fasting subjects in the morning. After centrifugation for $10 \mathrm{~min}$ at $1467 \mathrm{RCF}$, the serum was extracted and stored at $-80^{\circ} \mathrm{C}$. sL-selectin serum levels were measured using the enzyme-linked immunosorbent assay (ELISA) method and the following kit: Human L- Selectin/CD62L (R\&D Systems, Minneapolis, USA). The tests were performed according to the manufacturer's specifications. The ELISA microplate reader from MRXe Dynex Technologies (Chantilly, USA) was used. Additionally, the following biochemical parameters were measured in the blood serum sample: uric acid, CRP (C-reactive protein), total cholesterol, LDL cholesterol (low density lipoprotein), HDL cholesterol (high density lipoprotein), and triglycerides.

\section{Statistical analysis}

Statistical analysis was performed using CSS STATISTICA software for Windows (v. 5.0). Spearman's r correlation coefficient was used to assess the relationship between 2 variables and the Mann-Whitney U test was used to compare values between the 2 groups. Differences between samples were considered significant at $\mathrm{p}<0.05$.

This work was approved by the institution's relevant ethics committee: the Commission of Bioethics at Wroclaw Medical University. We obtained written informed consent from all participants involved in our study. 
Table 1. Selected parameters in examined group

\begin{tabular}{|l|c|c|c|}
\multicolumn{1}{|c|}{ Factor } & $\begin{array}{c}\text { Control group } \\
n=59\end{array}$ & $\begin{array}{c}\text { OSA patients } \\
n=163\end{array}$ & $\begin{array}{c}\text { Mann-Whitney } \\
\text { U test } \\
\text { p-value }\end{array}$ \\
\hline Age (years) & $51.27 \pm 12.97$ & $55.41 \pm 8.63$ & ns \\
\hline AHI/h & $2.21 \pm 1.90$ & $35.02 \pm 22.28$ & $<0.05$ \\
\hline DI/h & $2.88 \pm 2.76$ & $33.64 \pm 24.22$ & $<0.05$ \\
\hline SL-selectin (ng/mL) & $1350.73 \pm 569.75$ & $1080.02 \pm 175.29$ & $<0.05$ \\
\hline BMI & $29.47 \pm 5.42$ & $34.98 \pm 7.55$ & $<0.05$ \\
\hline CRP (mg/L) & $3.7 \pm 2.89$ & $6.55 \pm 6.24$ & ns \\
\hline Total cholesterol (mmol/L) & $5.52 \pm 1.12$ & $5.38 \pm 1.17$ & ns \\
\hline LDL cholesterol (mmol/L) & $3.39 \pm 0.93$ & $3.47 \pm 1.18$ & ns \\
\hline HDL cholesterol (mmol/L) & $1.25 \pm 0.34$ & $1.18 \pm 0.44$ & ns \\
\hline Triglicerids (mmol/L) & $2.04 \pm 1.38$ & $2.10 \pm 1.22$ & \\
\hline
\end{tabular}

AHI - apnea hypopnea index; DI - desaturation index; BMI - body mass index; CRP - C-reactive protein; LDL - low density lipoprotein cholesterol; HDL - high density lipoprotein cholesterol.

thors also observed a gradual increase in the plasma concentration of L-selectin as the severity of the OSA worsened. ${ }^{11}$

However other studies didn't show any differences in sL-selectin levels in sleep apnea syndrome. El-Solh et al. examined 4 adhesion molecules: ICAM-1 (intercellular adhesion molecule), VCAM-1 (vascular cell adhesion molecule), L-selectin and E-selectin in 15 patients with coronary artery disease (CAD) and moderate-to-severe OSA. All but the L-selectin molecules were significantly higher in OSA patients compared to the control group. ${ }^{5}$ Chinese authors demonstrated similar results evaluating the serum levels of adhesion molecules in OSA patients with and without hypertension. Serum levels of ICAM-1and VCAM-1 were significantly higher in OSA patients with or without hypertension than in the control group. However serum levels of L-selectin did not differ in the groups examined. ${ }^{12}$

Our study showed that sL-selectin se-

\section{Results}

sL-selectin serum levels were significantly lower in OSA patients than in the control group (1080.02 \pm 175.29 vs $1350.73 \pm 569.75 \mathrm{ng} / \mathrm{mL}, \mathrm{p}<0.05$ ) (Table 1 ). In addition, sL-selectin levels correlated negatively with AHI $\left(\mathrm{r}_{\mathrm{s}}=-0.162 ; \mathrm{p}<0.05\right)$ and DI $\left(\mathrm{r}_{\mathrm{s}}=-0.134 ; \mathrm{p}<0.05\right)$ while there was a positive relationship between sL-selectin levels and mean $\left(\mathrm{r}_{\mathrm{s}}=0.201 ; \mathrm{p}<0.05\right)$ and minimum $\left(\mathrm{r}_{\mathrm{s}}=0.157 ; \mathrm{p}<0.05\right)$ saturation.

sL-selectin levels were also lower in OSA patients with cardiovascular diseases compared with OSA patients without co-morbidities $(1065.91 \pm 179.30$ vs $1120.66 \pm 158.22$ $\mathrm{ng} / \mathrm{mL}, \mathrm{p}<0.05)$.

Spearman's r correlation coefficient showed that sL-selectin correlated positively with HDL-cholesterol $\left(r_{\mathrm{s}}=0.144\right.$; $\mathrm{p}<0.05)$ and negatively with uric acid $\left(\mathrm{r}_{\mathrm{s}}=-0.205 ; \mathrm{p}<0.05\right)$ and CRP $\left(\mathrm{r}_{\mathrm{s}=}-0.188 ; \mathrm{p}<0.05\right)($ Table 2$)$.

\section{Discussion}

The role of sL-selectin in sleep apnea syndrome is disputable and still not properly understood. We were only able to find a few studies on sL-selectin in OSA. Ohga et al. showed, in a relatively small group (6 OSA patients and 7 from the control group), that sL-selectin levels were higher in OSA patients compared to the levels in normal subjects. These authors also observed that sleep did not have an influence on sL-selectin levels in either the control group or OSA patients. ${ }^{10}$ Cofta et al. demonstrated similar results in a larger group (80 patients). These au- rum levels were significantly lower in OSA patients compared to the control group and that sL-selectin correlated negatively with OSA parameters such as AHI and DI. We found these results surprising as, to our knowledge, this

Table 2. The relationships between sL-selectin serum levels and selected parameters

\begin{tabular}{|l|c|c|}
\multirow{2}{*}{ Factor } & \multicolumn{2}{|c|}{ sL-selectin serum levels } \\
\cline { 2 - 3 } & \multicolumn{1}{|c|}{$\mathrm{r}_{\mathrm{s}}$} & $\mathrm{p}$-value \\
\hline Age & -0.161 & $<0.05$ \\
\hline AHI & -0.162 & $<0.05$ \\
\hline DI & -0.134 & $<0.05$ \\
\hline BMI & -0.066 & $\mathrm{~ns}$ \\
\hline CRP & -0.188 & $<0.05$ \\
\hline Total cholesterol & 0.036 & $\mathrm{~ns}$ \\
\hline LDL cholesterol & 0.014 & $\mathrm{~ns}$ \\
\hline HDL cholesterol & 0.144 & $<0.05$ \\
\hline Triglicerids & -0.099 & $<0.05$ \\
\hline Mean saturation & 0.157 & $<0.05$ \\
\hline Minimum saturation & -0.205 & $<0.05$ \\
\hline Uric acid & 0.201 & $<0.05$ \\
\hline
\end{tabular}

AHI - apnea hypopnea index; DI - desaturation index; BMI - body mass index; CRP - C-reactive protein, LDL - low density lipoprotein cholesterol; $\mathrm{HDL}$ - high density lipoprotein cholesterol. 
is the first study suggesting that sL-selectin may be lower in OSA patients and that this could correlate negatively with OSA parameters. These results could be linked to the high incidence of cardiovascular disorders in the group examined. However they may also change our point of view on the role of sL-selectin in OSA, especially given that we also demonstrated that sL-selectin levels were lower in OSA patients with cardiovascular diseases than in those without co-morbidities.

Decreased sL-selectin levels have been described in many cardiovascular disorders as well as after trauma. In type 2 diabetes, sL-selectin is not only decreased, but vascular complications are accompanied by a decrease in the leukocyte surface expression of L-selectin. ${ }^{13}$ Serum levels of sL-selectin are lower in acute ischemic stroke compared with higher levels of other adhesion molecules such as ICAM (intercellular adhesion molecule), VCAM (vascular cell adhesion molecule) and E-selectin. ${ }^{14}$ After severe trauma, a decrease in sL-selectin indicates an increased likelihood of lung failure and multiorgan dysfunction syndrome. ${ }^{15}$ Different studies have demonstrated that sL-selectin levels were lower in patients with stable angina, unstable angina and acute myocardial infarction and in patients with coronary artery disease and type II diabetes. ${ }^{16,17}$ Some authors even suggested that a marked fall in sL-selectin might constitute a marker for silent CAD (coronary artery disease) in patients with type 2 diabetes. ${ }^{17}$ Other studies indicate that the stimulation of leukocytes in patients with ischemic heart disease leads to down regulation of surface L-selectin expression, which results in a decrease in levels of circulating sL-selectin. ${ }^{16}$ In addition, the findings by Rozenberg et al. in an animal model suggest that $\mathrm{L}$-selectin could have a protective role against atherosclerosis. ${ }^{18}$ In addition, we showed that sL-selectin correlated positively with HDLcholesterol and negatively with uric acid and CRP. These correlations may also suggest the anti-atherosclerotic effect of sL-selectin. We also demonstrated that CRP was significantly higher in OSA patients $(\mathrm{p}<0.05)$. Our findings are in agreement with many previous studies, having demonstrated that the activation of an inflammatory process plays an important role in sleep apnea syndrome and in atheromatosis development. ${ }^{19,20}$ In several studies, CRP was examined along with other inflammatory markers such as TNF-alpha (tumor necrosis factor), fibrinogen, interleukins IL-6 and IL-8, ICAM and VCAM. ${ }^{21}$

More recent studies also indicate that hyperuricemia could increase the risk of cardiovascular diseases and their complications, probably through the influence on endothelial dysfunction, oxidative stress and inflammation. $^{22}$

We realize that our study has a few limitations. First of all, there are differences in BMI between the OSA patients and control group and sL-selectin correlated negatively with age. The relationship between age and Lselectin is not clear. Some authors have suggested, that
L-selectin levels could decrease until 55 years old. ${ }^{23}$ In addition, the age-related differences in other inflammatory markers have been described. This could be connected with changes in visceral adipose tissue accumulation. ${ }^{24}$ Moreover, such a high percentage of OSA patients with cardiovascular diseases (75\%) could have an influence on the proper interpretations of the results. It is difficult to assess whether the low serum level of sL-selectin was associated only with OSA or cardiovascular disease (or both entities), especially since the correlations demonstrated were weak (even though statistically significant). An additional analysis in patients with cardiovascular diseases but without OSA could be helpful in the better understanding of the sL-selectin changes observed. However, we did not include this group of patients. We also have to remember that the patients examined received drugs from different groups as statins, ACEIs or beta-blockers. We did not find in the literature any studies describing drug influences on sL-selectin serum levels, but experimental studies suggest that cholesterol lowering therapy in mononuclear cells from hypercholesterolemic patients could reduce mRNA and protein expression of L-selectin and other adhesion molecules. ${ }^{25}$

\section{Conclusions}

Our work, together with observations relating to other diseases and experimental studies, suggests that decreased sL-selectin levels could play a role in an increased risk of cardiovascular complications in sleep apnea syndrome. However future studies are needed to understand the role of sL-selectin in sleep apnea syndrome.

\section{References}

1. Lurie A. Metabolic disorders associated with obstructive sleep apnea in adults. Adv Cardiol. 2011;46:67-138.

2. McNicholas WT, Bonsigore MR, Management Committee of EU COST ACTION B26. Sleep apnoea as an independent risk factor for cardiovascular disease: Current evidence, basic mechanisms and research priorities. Eur Respir J. 2007;29(1):156-178.

3. Akinnusi ME, Paasch LL, Szarpa KR, Wallace PK, El Solh AA. Impact of nasal continuous positive airway pressure therapy on markers of platelet activation in patients with obstructive sleep apnea. Respiration. 2009;77(1):25-31.

4. Lavie L, Lavie P. Molecular mechanisms of cardiovascular disease in OSAHS: the oxidative stress link. Eur Respir J. 2009;33(6):1467-1484.

5. El-Solh AA, Mador MJ, Sikka P, Dhillon RS, Amsterdam D, Grant BJ. Adhesion molecules in patients with coronary artery disease and moderate-to-severe obstructive sleep apnea. Chest.2002;121(5):1541-1547.

6. Barkhausen T, Krettek C, van Griensven M. L-selectin. Adhesion, signaling and its importance in pathologic posttraumatic endotoxemia and non-septic inflammation. Exp Toxicol Pathol. 2005;57(1):39-52.

7. Tedder TF, Steeber DA, Chen A, Engel P. The selectins: Vascular adhesion molecules. FASEB J. 1995;9(10):866-873.

8. Wedepohl S, Beceren-Braun F, Riese S, et al. L-selectin - a dynamic regulator of leukocyte migration. Eur J Cell Biol. 2012;91(4):257-264.

9. Schleiffenbaum B, Spertini O, Tedder TF. Soluble L-selectin is present in human plasma at high levels and retains functional activity. J Cell Biol. 1992;119(1):229-238. 
10. Ohga E, Nagase T, Tomita T, et al. Increased levels of circulating ICAM-1, VCAM-1, and L-selectin in obstructive sleep apnea syndrome. J Appl Physiol. 1999;87(1):10-14.

11. Cofta S, Wysocka E, Dziegielewska-Gesiak S, et al. Plasma selectins in patients with obstructive sleep apnea. Adv Exp Med Biol. 2013;756:113-119.

12. Li YZ, Zhang WR, Wang TC, Lu HX, Wang Y, Wang X. The role of adhesion molecules in the pathogenic mechanisms of hypertension in obstructive sleep apnea-hypopnea syndrome. Zhonghua Jie He He Hu Xi Za Zhi. 2004;27(8):511-514.

13. Mastej K, Adamiec R. Serum level of sL-selectin and leukocyte surface expression of L-selectin in patients with type 2 diabetes. Przegl Lek. 2011;68(3):140-145.

14. Simundic AM, Basic V, Topic E, et al. Soluble adhesion molecules in acute ischemic stroke. Clin Invest Med. 2004;27(2):86-92.

15. Stengel D, Bauwens K, Keh D, et al. Prognostic value of an early soluble L-selectin (sCD62L) assay for risk assessment in blunt multiple trauma: A meta-analysis. Clin Chem. 2005;51(1):16-24.

16. Haught WH, Mansour M, Rothlein R, et al. Alternations in circulating intercellular adhesion molecule-1 and L-selectin: Further evidence for chronic inflammation in ischemic heart disease. Am Heart $J$. 1996;132(1):1-8.

17. Albertini JP, Valensi P, Lormeau B, Vaysse J, Attali JR, Gattegno. Soluble L-selectin level is a marker for coronary artery disease in type 2 diabetic patients. Diabetes Care. 1999;22(12):2044-2048.
18. Rozenberg I, Sluka SH, Mocharla P, et al. Deletion of L-selectin increases atherosclerosis development in ApoE-/-mice. PLoSOne. 2011;6(7):e21675

19. Jelic S, Le Jemtel TH. Inflammation, oxidative stress, and the vascular endothelium in obstructive sleep apnea. Trends Cardiovasc Med. 2008;18(7):253-260.

20. Libby P, Okamoto $Y$, Rocha VZ, Folco E. Inflammation in atherosclerosis: Transition from theory to practice. Circ J. 2010;74(2):213-220.

21. Kanbay M, Segal M, Afsar B, Kang DH, Rodriguez-Iturbe B, Johnson RJ. The role of uric acid in the pathogenesis of human cardiovascular disease. Heart. 2013; 99(11):759-766.

22. Nadeem R, Molnar J, Madbouly EM, et al. Serum inflammatory markers in obstructive sleep apnea: A meta-analysis. J Clin Sleep Med. 2013;15(9):1003-1012.

23. Ponthieux A, Herbeth B, Droesch S, Haddy N, Lambert D, Visvikis S. Biological determinants of serum ICAM-1, E-selectin, P-selectin and L-selectin levels in healthy subjects: The Stanislas study. Atherosclerosis. 2004;172(2):299-308.

24. Cartier A, Cote M, Lemieux I, et al. Age-related differences in inflammatory markers in men: Contribution of visceral adiposity. Metabolism. 2009;58(10):1452-1458.

25. Cerda A, Rodrigues AC, Alves $C$, et al. Modulation of adhesion molecules by cholesterol-lowering therapy in mononuclear cells from hypercholesterolemic patients. Cardiovasc Ther. 2015;33(4):168-176. 